\title{
Pen + Touch Diagramming to Stimulate Design Ideation
}

\author{
Andrew M. Webb \\ Interface Ecology Lab \\ Department of Computer Science and Engineering \\ Texas A\&M University \\ andrew@ecologylab.net
}

\begin{abstract}
Ideation, the process of generating new ideas, is central to design where the goal is to find novel solutions around a set of requirements. Designers engage in diagramming, creating external representations of ideas. Diagramming connects the body and creative cognitive processes, as the hands transform physical media to explore ideas. HCI researchers need to leverage body-based interaction to support creativity. My $\mathrm{Ph}$.D. research develops a new body-based diagramming environment to stimulate design ideation.
\end{abstract}

\section{Author Keywords}

diagramming, design, ideation, body-based interaction

\section{ACM Classification Keywords}

H.5.1 Information interfaces and presentation: HCI.

\section{INTRODUCTION}

Generating new ideas is critical for design. Design processes are supported by embodied representations, including gestures, tangibles, and diagrams, which have been found to help people think $[9,8]$. A diagram is a design thinking tool that stimulates imagination [1]. The process of diagramming mediates exploration of relationships between concepts, using ambiguity to foster multiple interpretations. Designers explore ideas by transforming physical diagrams (e.g. cutting, folding, sketching over) using their hands (with tools). The proliferation of sensory modalities, such as multi-touch, pen, and computer vision, enables cost-effective development of new forms of body-based interaction that can support expressive diagramming. We hypothesize that making diagramming more based in the body will promote exploration of diverse transformations, stimulating ideation.

We develop a new diagramming environment to investigate how body-based interactions impact creative cognitive processes. We derive new bimanual pen + touch interactions for diagramming on personal surfaces. We evaluate our new environment in landscape architecture education.

Permission to make digital or hard copies of part or all of this work for personal or classroom use is granted without fee provided that copies are not made or distributed for profit or commercial advantage and that copies bear this notice and the full citation on the first page. Copyrights for third-party components of this work must be honored. For all other uses, contact the Owner/Author.

Copyright is held by the owner/author(s)

C\&C '15, June 22-25, 2015, Glasgow, United Kingdom

ACM 978-1-4503-3598-0/15/06.

http://dx.doi.org/10.1145/2757226.2764766

\section{BACKGROUND}

We ground design of our environment in embodied cognition. Cognitive models, including those associated with creativity, are based in the body [2]. Tversky et al. demonstrate that gestures help people not just to communicate meaning, but further, to remember and to understand complex ideas [10]. Of particular value are iconic gestures, whose shapes map directly to what they mean, metaphoric gestures, that use spatial representation to convey relationships, and embodied gestures, which encode knowledge motorically, as images and diagrams encode pictorially. These species of gestures provide us with cognitive guidance for how to develop body-based interfaces that will help designers diagram and form new ideas.

We seek to embody gestural interaction with diagrams through bimanual pen + touch techniques. Guiard developed one of the first models of bimanual interaction, the kinematic chain [5]. In a kinematic chain, the non-preferred hand (NPH) acts to define a reference frame for the actions of the preferred hand $(\mathrm{PH})$. When drawing on paper, this is equivalent to the $\mathrm{NPH}$ positioning and rotating the paper in conjunction with the PH making marks with a pen. We design pen + touch interaction techniques using the kinematic chain model.

\section{DIAGRAMMING ENVIRONMENT}

We are developing a new diagramming environment to investigate how bimanual pen + touch interactions can support design processes. Through iterative design, we are evaluating impact on design ideation in architecture education.

\section{Information Composition + Sketching}

Our diagramming environment integrates information composition with sketching in an infinite zoomable space. Information composition, a medium for representing a personal information collection as a connected whole, supports reflection when performing information-based ideation tasks [11] Designers engage in information-based ideation tasks, using information as support for generating new ideas [7], such as investigating how properties of different materials will impact a design. In our diagramming environment, designers gather images and text while sketching out ideas and exploring relationships amidst the collected information (see Figure 1).

Sketches act as interactive imagery [3]. Strokes are drawn, re-drawn, drawn over, and erased, transforming ideas. Design ideation environments should support abstraction, ambiguity, and imprecision in sketching [4]. Through integrating information composition with sketching, we support ambiguity and varied interpretations in diagramming. 


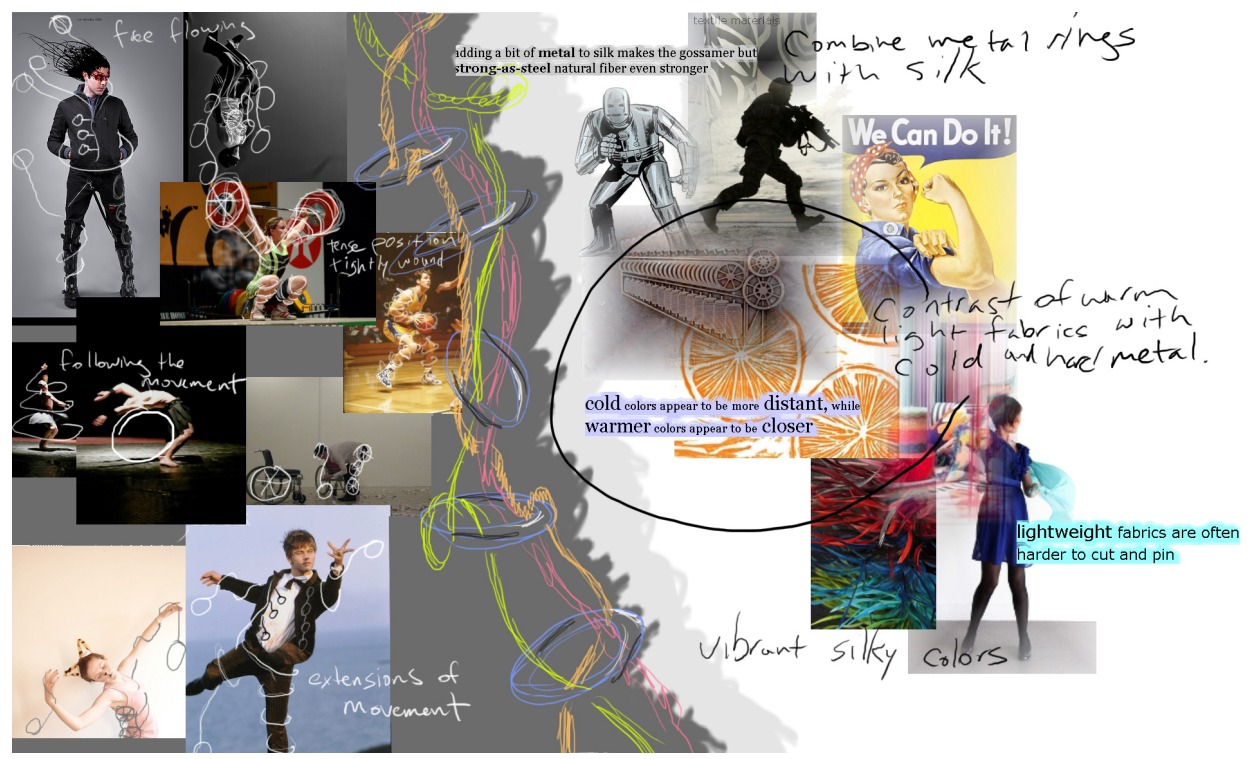

Figure 1: Example information composition + sketching diagram created by a hypothetical student for a fashion design assignment. Student searched for information on materials, such as textiles, and collecting resources. She developed idea of warm vibrant silk contrasting with cold dark metal (right). Circle shapes inspired design of metal rings with silk strands. Ideas were explored by sketching over these images.

created, video recorded their body-based interactions while working in the environment, and interviewed several participants. We are in the process of analyzing this data, both in terms of quantitative ideation metrics [7] and qualitative coding of video and interview data.

\section{CONCLUSION}

HCI researchers have the opportunity to transform design processes with the development of new body-based interactions. Digital environments enable creation and exploration of large information spaces that can be dynamically transformed. Interactions with these environments need to be expressive to support diverse transformations. Simultaneous pen and multi-touch input will support such expressive transformations. Developing pen + touch interaction techniques that use kinematic chains

\section{Body-based Diagramming: Pen + Touch Interaction}

We seek to provide pen + touch interactions that are intuitive, expressive, and more directly connect designers to diagrams. The goal is not simply to make diagramming easier, but to aid designers in forming abstractions and investigating ambiguous representations through body-based experiences. While diagrams vividly convey spatial relationships and ideas, much of the thinking and mental models of the author are encoded in body-based creative processes of transformation. We define diagram transformation as any operation that changes a diagram to encode meaning, e.g.: adding and removing elements, affine transforms, color-space transforms, cropping, and distortion. Just as a designer uses her hands to transform physical diagrams (e.g. rotating, folding, or bending material), body-based interaction techniques are needed for transforming our new diagramming medium.

As recommended by Hinckley et al. [6], the pen, when used by itself, makes marks. The exception is when kinematic chains are created. When pen input is combined with touches, that act as modifiers, commands are invoked. We develop new bimanual pen + touch interaction techniques for diagram transformation, including cropping, layering, rotating with 3D perspective, and brush styling. Designers fluidly switch between sketching ideas and transforming diagram elements. Designers investigate varied ideas through transformations, while using a NPH undo gesture to iteratively revert changes.

\section{Evaluation: Landscape Architecture Education}

As a context for evaluating effects of body-based diagramming on design ideation, we are engaging in field studies in architecture education. Student teams in two design studios have used a preliminary version of our diagramming environment on course assignments. We collected the diagrams they will help designers think about how elements are transformed and the relationships between elements and transformations.

\section{REFERENCES}

1. Deleuze, G., and Guattari, F. A Thousand Plateaus: Capitalism and Schizophrenia. University of Minnesota Press, 1987.

2. Glenberg, A. Why Mental Models Must Be Embodied. Advances in Psychology 128 (1999), 77-90.

3. Goldschmidt, G. The Dialectics of Sketching. Creativity Research Journal 4, 2 (1991), 123-143.

4. Gross, M. D., and Do, E. Y.-L. Ambiguous intentions: a paper-like interface for creative design. In Proc. UIST (1996), 183-192.

5. Guiard, Y. Asymmetric division of labor in human skilled bimanual action: The kinematic chain as a model. Journal of motor behavior 19 (1987).

6. Hinckley, K., Yatani, K., Pahud, M., Coddington, N., Rodenhouse, J., Wilson, A., Benko, H., and Buxton, B. Pen + touch $=$ new tools. In Proc. UIST (2010).

7. Kerne, A., Webb, A. M., Smith, S. M., Linder, R., Lupfer, N., Qu, Y., Moeller, J., and Damaraju, S. Using metrics of curation to evaluate information-based ideation. ACM Trans. Comput.-Hum. Interact. 21, 3 (June 2014), 14:1-14:48.

8. Kim, M., and Maher, M. Comparison of designers using a tangible user interface $\&$ graphical user interface and impact on spatial cognition. In Proc. Human Behaviour in Design (2005).

9. Suwa, M., and Tversky, B. What do architects and students perceive in their design sketches? a protocol analysis. Design Studies 18, 4 (1997), $385-403$

10. Tversky, B., Heiser, J., Lee, P. U., and Daniel, M. P. Explanations in gesture, diagram, and word. Oxford University Press, Oxford, 2009, 119-131.

11. Webb, A. M., Linder, R., Kerne, A., Lupfer, N., Qu, Y., Poffenberger, B., and Revia, C. Promoting reflection and interpretation in education: Curating rich bookmarks as information composition. In Proc. Creativity and cognition (2013). 